Acta Cryst. (1953). 6, 821

\title{
Unusual X-ray Diffraction Effects from a Crystal of Wollastonite
}

\author{
By J. W. JEFFERY \\ Crystallographic Laboratory, Birkbeck College, Malet Street, London W.C. 1, England
}

(Received 6 February 1953 and in revised form 20 May 1953)

\begin{abstract}
Crystals of wollastonite from Devon are shown to be composites of monoclinic and triclinic forms. Streaks in the $a^{*}$ direction on odd layer Weissenberg photographs show the presence of very thin plates parallel to $b c$. The changes from one lattice to another correspond to 'mistakes' of $\frac{1}{2} b$ in packing. The occurrence of these mistakes is explained in terms of the structure. The alternative descriptions of the occurrence of the two triclinic forms in terms of twinning are given, and related to the theory of twin formation. Space-group twinning is discussed in relation to the polymorphism of wollastonite.
\end{abstract}

As a result of the $\mathrm{X}$-ray investigation of Warren \& Biscoe (1931) and the exhaustive optical and goniometric study by Peacock (1935a), wollastonite $\left(\mathrm{CaSiO}_{3}\right)$ is now known to exist in two low-temperature forms. These forms are so similar as to be almost indistinguishable optically and they were both originally referred to the same set of monoclinic morphological axes. The triclinic form, or wollastonite proper, is found in massive deposits of metamorphosed limestone and has cell dimensions

$$
\begin{gathered}
a=7 \cdot 88, b=7 \cdot 27, c=7 \cdot 03 \AA ; \\
\alpha=90^{\circ}, \beta=95^{\circ} 16^{\prime}, \gamma=103^{\circ} 22^{\prime}
\end{gathered}
$$

(Warren \& Biscoe, 1931 ; Peacock, 1935a). The monoclinic form, or parawollastonite, is found chiefly in limestone blocks ejected from volcanoes and its cell dimensions are

$$
\begin{gathered}
a=15 \cdot 33, b=7 \cdot 28, c=7 \cdot 07 \AA ; \\
\alpha=\gamma=90^{\circ}, \beta=95^{\circ} 24 \frac{1}{2}^{\prime}
\end{gathered}
$$

(Barnick, 1936). The differences in the lengths of the $b$ and $c$ axes of the two forms are probably due to

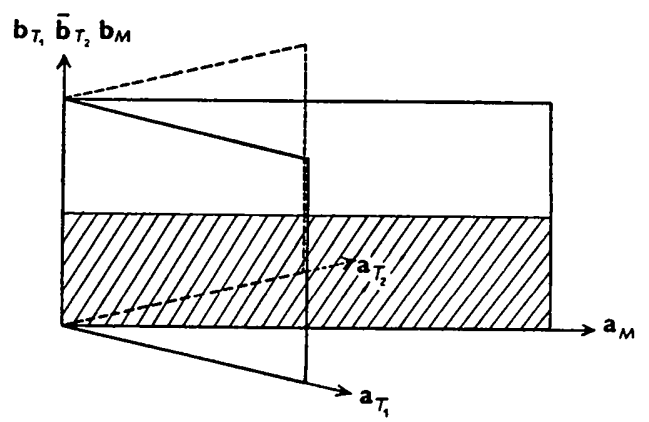

Fig. 1. The outline in the $a b$ plane of the unit cells of monoclinic $(M)$ and right-handed $\left(T_{1}\right)$ and left-handed $\left(T_{2}\right)$ triclinic wollastonite, in the orientations in which they occur in the composite crystal. The $c$-axis direction is the same for all three cells and comes up out of the paper slightly inclined to the left. The monoclinic pseudo-cell is shown shaded. experimental error; the accurate goniometric study of Peacock gives the same $c / b$ ratio for the two forms, and X-ray photographs of a composite crystal show no separation of the common set of $h 0 l$ reflexions, or of layer lines about $b$. In both cases the values of $b$ and $c$ were derived from layer-line measurements. Taking values which satisfy the morphological $c / b$ ratio, it can be said that both forms have

$$
b=7 \cdot 28, c=7.03 \AA \text {, and } \alpha=90^{\circ} .
$$

The relationship between the unit cells is shown in Fig. 1. The transformation between monoclinic and triclinic (subscripts $M$ and $T$ respectively) is given by the following vector relations:

$$
\mathbf{a}_{T}=\frac{1}{2} \mathbf{a}_{M}-\frac{1}{4} \mathbf{b}_{M} ; \mathbf{b}_{T}=\mathbf{b}_{M} ; \mathbf{c}_{T}=\mathbf{c}_{M} \text {. }
$$

Relations with identical coefficients hold for $h k l$. It follows that the triclinic [410] direction is the same as that of the monoclinic $a$ axis, and this accounts for the early confusion of the two forms.

There is no detectable difference between the two forms in their even layer lines about $b$; only the odd layer lines show lack of a plane of symmetry in the triclinic form. These odd layers are very weak in both forms. It is thus useful to define a pseudo-cell with $a$ and $b$ parallel to those of the monoclinic cell, but with $b$ halved. This cell is shown in Fig. 1 .

The wollastonite used in the present investigation came from Devon and was very fibrous in character. It cleaved very easily parallel to the fibre direction and fragments obtained by crushing or cutting across the fibre tended to be very 'ragged', or to be partially cleaved and therefore containing two or more individuals slightly misaligned. A large number of fragments were examined by $\mathrm{X}$-rays before a clean single individual was found. Oscillation photographs about $b$ (the fibre direction) apparently showed monoclinic symmetry, but in all cases streaks parallel to the layer lines occurred in the odd layers. Weissenberg photographs of the even layers about $b$ (Fig. 2) show that these reflexions can be indexed using the pseudo- 


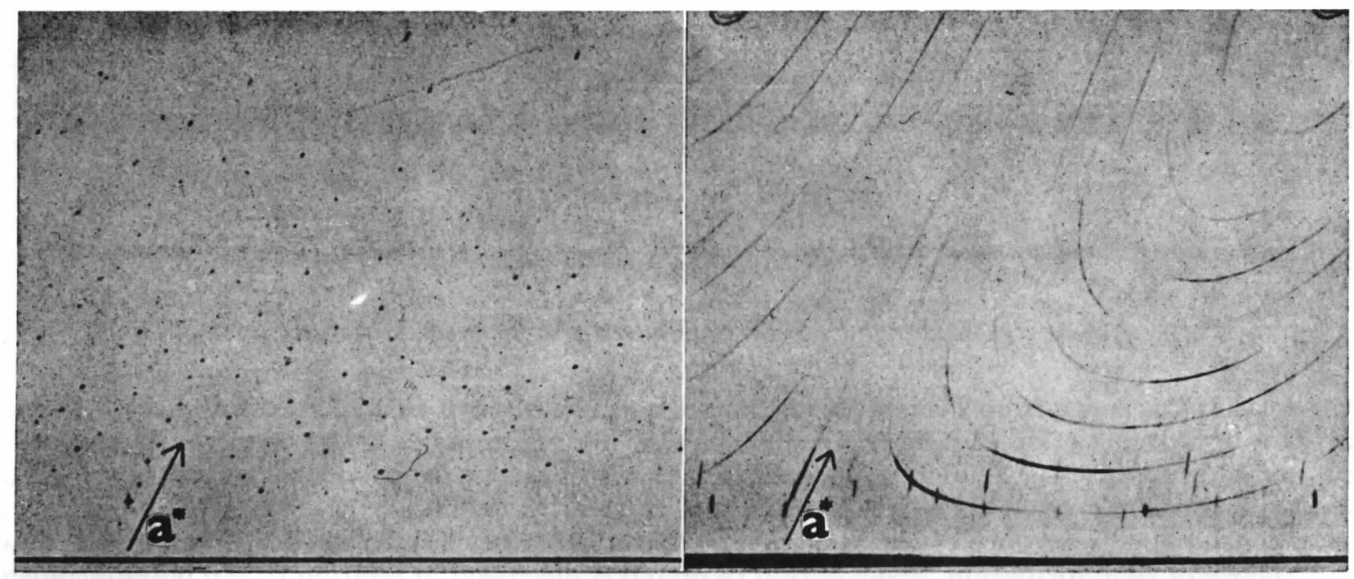

Fig. 2.

Fig. 3.

Fig. 2. Superimposed zero- and 2nd-layer equi-inclination Weissenberg photographs about $b$. The photographs, which necessarily have a slightly different scale, have also been given a slight relative displacement sideways so that the two sets of reflexions can be more readily distinguished. The fourth layer has reflexions in the same positions as the zero layer. The face-centering is most clearly seen along and to the left of $a^{*}$.

Fig. 3. lst-layer equi-inclination Weissenberg photograph about $b$.

lattice defined above, and that this lattice is facecentred on $C$; the absences are of the form $2 h+k=$ $2+4 n$ if the indices refer to the true (monoclinic) cell. Even-layer Weissenberg photographs were taken of a number of crystals, and, apart from elongation of the spots due to the presence of several slightly misoriented individuals, no abnormality could be detected; in particular no streaks were observed.

Fig. 3 shows the first-layer Weissenberg photograph of the perfect fragment. Photographs of other fragments were similar, all having streaks connecting the spots in the $a^{*}$ direction, but in some cases rather diffuse spots could be seen on the streaks half way between the spots shown in Fig. 3. These more diffuse spots correspond to the monoclinic form of wollastonite and the others to the presence of both 'right-handed' and 'left-handed' triclinic lattice orientations. ('Righthanded' and 'left-handed' are used only to denote the two sets of axes and imply nothing about the structure, which is, in fact, centrosymmetrical.)

Streaks have also been observed in a number of photographs of specimens of wollastonite from different sources, so that the phenomenon must be quite common.

The fact that streaks occur in the $a^{*}$ direction shows the presence of plates only a few unit cells thick in this direction (Ewald, 1940). Since the monoclinic reflexions, when they occur, are the more diffuse it is probable that the thinnest plates have this lattice (in some cases the plates are apparently so thin that no maximum can be detected on the streak), but there must be a fairly rapid alternation of the three lattices throughout the crystal. Streaks do not occur in the even layers, and therefore such reflexions for all three lattices must be in phase. In other words, the 'mistakes' in position giving rise to the alternating lattices must be exactly equal to $\frac{1}{2} b^{*} . \dagger$ It is of interest to see if the structure can throw some light on the reason for this lattice alternation.

\section{The structure of wollastonite}

Barnick (1936) derived the atomic arrangement of parawollastonite, but his structure has been criticized, mainly on the grounds that it does not adequately explain the fibrous habit or the cleavage directions. It can also be criticized, as can Ito's (1950) verification of the triclinic structure derived from it, on the grounds of the poor agreement of observed and calculated X-ray intensities. A redetermination of the structure is in progress in this laboratory, but fortunately for the present purpose it is sufficient to establish the symmetry and some parameters of the 'asymmetric unit' in the true cell. This can be done by utilising the exact $C$ face-centring of the pseudo-cell, and the symmetry elements of the true cell, which has the space group $P 2_{1} / a$. Fig. $4(a)$ shows the true cell, divided by the plane $y=\frac{1}{2}$ into two pseudo-cells, each of which must be face-centred. (The theoretically possible alternative, that the scattering from the two halves of the true cell is of equal amplitude but opposite phase for the absent reflexions, can be ruled out since there are more independent absences than independent parameters in the structure.) The two pseudo-cells are related by the screw axes and the two units in each produced by the face-centring are also related by reflexion across the glide plane at $y=\frac{1}{4}\left(\frac{3}{4}\right)$. They must therefore lie on the planes $y=\frac{1}{8}, \frac{3}{8}$ (and $\left.\frac{5}{8}, \frac{7}{8}\right)$ and be symmetrical about these planes. This is

$\dagger$ This is the 'hypothetical case' analysed in detail by Wilson (1949). 


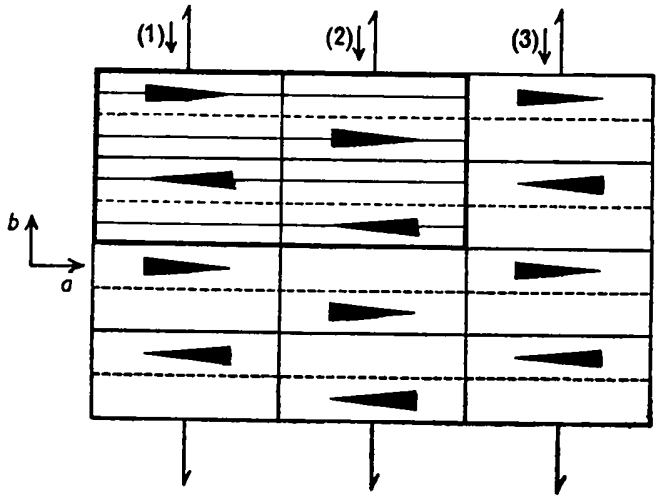

(a)

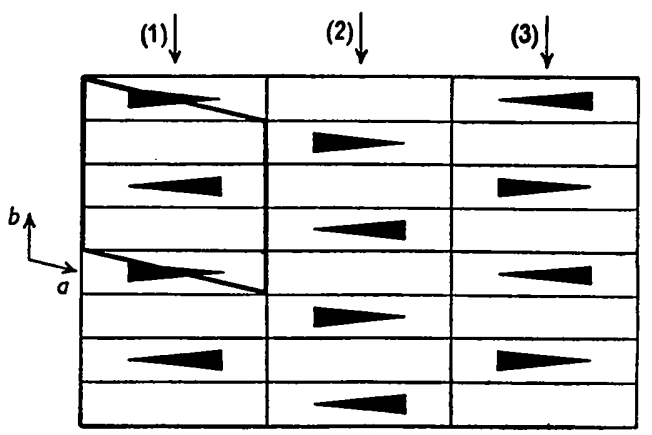

(b)

Fig. 4. Section in the $a b$ plane through the layers parallel to $b c$ (marked (1), (2), (3) and coming, slightly inclined to the left, out of the paper). (a) Monoclinic lattice. The stacking of the layers takes place with an alternating shift of $\frac{1}{4} b$. $(b)$ Triclinic lattice. The stacking of the layers takes place with a progressive shift of $\frac{1}{4} b$. The unit cells are heavily outlined in each case.

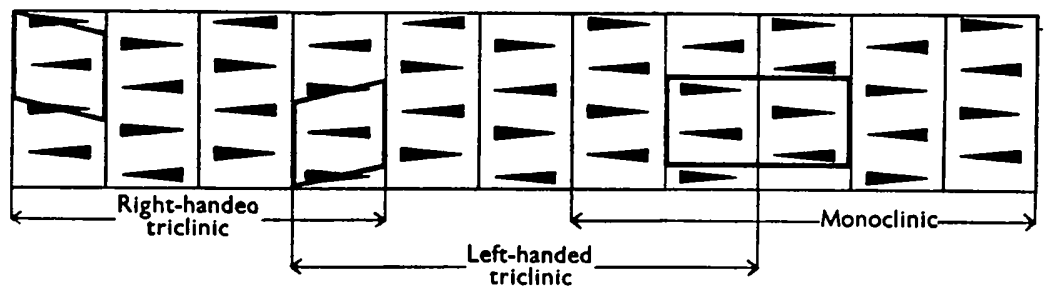

Fig. 5. Diagram showing how the three methods of stacking (monoclinic, right- and left-handed triclinic) can alternate through the crystal.

the condition derived analytically from the non-spacegroup absences for $2 h+k=2+4 n$, by Barnick (1936) and, contrary to the statement by Ito (1950), explicitly recognized by him. We can therefore represent the 'asymmetric unit' by a wedge-shaped symbol, as in Fig. 4.

In the layers of wedges parallel to the $b c$ plane the asymmetric units are arranged under the influence of nearest neighbours and no possibility of 'mistakes' arises. But in the monoclinic $a$ direction this is not the case. Fig. 4 shows a section through the first three layers in this direction, for the monoclinic case $(a)$ and the triclinic case $(b)$. The second layer is shown in one of the two possible positions (the other position is illustrated by layer 3 in relation to 2 in Fig. $4(a)$, but this alternative position is equivalent to moving the origin in the monoclinic case and taking lefthanded instead of right-handed axes for the triclinic lattice, so that it need not be considered separately). The third layer can then occupy two possible positions, one of which (Fig. 4(a)) builds a monoclinic lattice and the other (Fig. $4(b)$ ) builds a triclinic lattice.

Since the units are symmetrical ${ }^{*}$ there can be no energy difference between the two possible arrangement of adjacent layers, and the main influence in

* This is strictly proved only for the monoclinic lattice, but any distortion in the triclinic case can only be small. deciding which arrangement is followed must be exerted by the next-but-one layer $7.7 \AA$ away. This would account for the fact that the energy difference between these two forms of wollastonite is so small that it is difficult even to be certain which of the two is the low-temperature form, let alone map their phase fields. It follows that if the temperature is near the inversion point for the two structures it is possible for 'mistakes' to occur during growth and for a crystal to be formed which is composed of alternating layers of monoclinic and the left- and right-handed triclinic forms, as shown diagrammatically in Fig. 5 .

There is some morphological evidence in support of this hypothesis. Parallel growths are of common occurrence in wollastonite, and Peacock (1935a) found that the considerable number of such monoclinic composites which he investigated all had (100) as contact plane. Two examples of composites of a monoclinic and a triclinic crystal were also in contact on the common (100) face. The latter case is clearly due to a 'mistake' occurring once and being continued. The individuals of the monoclinic composites are probably separated by a very thin triclinic layer which is just sufficient to prevent the propagation of monoclinic faces across it, but too thin to be otherwise detected. In other words, it corresponds to a 'mistake' in growth which is corrected shortly afterwards. 


\section{The relation between the two triclinic forms}

If the $b c$ layers are assumed to be the same whether stacked to form a monoclinic or a triclinic lattice, then it follows from: (i) the triclinic crystal class, $(\overline{1})$; (ii) the non-space-group symmetry plane perpendicular to $b[010]$ contained in the structural unit; (iii) the fact that in the triclinic lattice $c$ and [410] are both perpendicular to $b$; that the two triclinic forms can be regarded either as inversions of one another or as twins on $(1 \overline{4} 0)$ or about $b[010]$. In no case do these operations produce one form as a direct continuation of the other. Even rotation about $b$ requires a translation in addition to produce a continuous series of layers.

The choice of inversion ('right- and left-handed') as the description of the relationship between the two forms in this paper was probably influenced by Peacock's (1935a) reference to the occurrence of uniterminal prisms which had either right-handed or lefthanded terminations. In considering the process of growth by the addition of layers, the change from one method of triclinic stacking to the other was thought of in terms of growth towards either a left-handed or right-handed termination. However, it can equally well be thought of as twinning on $(1 \overline{4} 0)$, or about $b[010]$, with (100) as composition plane in each case.

If the change is regarded as twin formation it does, in fact, illustrate very clearly some of Buerger's (1945) ideas on the genesis of twin crystals. Thus: (a) The structure permits a continuation of itself in alternative twin-junction configuration without alteration of the coordination of the atoms at the junction for a radius of $7 \AA$, and the junction therefore has very low energy. (b) The appearance of the bulk sample of Devon wollastonite and its excessively easy cleavage suggest that some chemical action has taken place preferentially at twin boundaries, enhancing the ease of parting. The differences in the ease of cleavage reported in the literature (Peacock, 1935a) may be due to a confusion between cleavage and parting on (100). (c) The twin boundary is necessarily a region in which at least three layers are arranged in monoclinic orientation, illustrating the general rule that the structure in a twin boundary is at least a possible polymorphous alternative structure.

Twinning about $b[010]$ has not previously been reported for triclinic wollastonite, the only known twinning being on (100). However, only one example of this is known and Peacock (1935a) conld not find a single example of twinned triclinic crystals in the very considerable amount of material available to him. The lack of morphological evidence of twinning is probably due to the occurrence of multiple twinning, as in the Devon wollastonite, if the conditions for twinning are present at all. It may be significant that pectolite, which is isomorphous with triclinic wollastonite, but with $\alpha$ greater than a right angle by $24 \frac{1}{2}^{\prime}$, was shown by Peacock (1935b) to twin about $b[010]$, whereas previously it had been thought to twin on (100).

\section{The nature of the asymmetric unit}

While the phenomena described above tell us nothing new about the structure of the asymmetric unit, it must be said that the $\mathrm{Si}_{3} \mathrm{O}_{9}$ ring postulated by Barnick (1936) has all the properties deduced above; and, in addition, two such rings related by a screw axis through the centre do fit together with normal inter-atomic distances at a spacing of $3 \cdot 6 \AA$. Any postulated chain system of $\mathrm{SiO}_{4}$ tetrahedra would probably have to be related to such a ring system in order to satisfy even the systematic absences of the diffraction data.

\section{Twinning polymorphism of wollastonite}

Ito (1950) has pointed out that when systematic absences occur which are not required by the space group, this is an indication of extra (virtual) symmetry elements operating partially or completely within each cell separately, but not throughout the lattice as a whole. The 'virtual' reflexion planes at $y=\frac{1}{8}$, etc. in parawollastonite are an example of this. Ito (1950) has elaborated a theory of twinned space groups to account for the relation between certain types of polymorphic structures, including those showing the presence of 'extra' symmetry elements. This theory may well be useful in suggesting a trial structure from space-group twinning relationships with a known structure. In the case of wollastonite, however, the relations which Ito deduces by means of this theory can be reached much more easily from the facecentring of the pseudo-cell of parawollastonite. In fact all the relations derived by transformations from the theory of twinned space groups can be written down by inspection from Fig. 4. The rather formal approach of his theory also leads Ito (1950) to postulate a third polymorph of wollastonite which he calls 'protowollastonite', and which has a unit cell equivalent to half the unit cell of parawollastonite (bounded by $x=\frac{1}{2}$, Fig. 4(a)). From this assumed unit cell, spacegroup twinning can produce the unit cells of both known forms of wollastonite. However, in a lattice composed of such cells the packing of the structural units would be entirely different from that of the known forms of wollastonite and there seems to be little justification for naming a new and as yet undiscovered mineral on the basis of this artificial construction.

This 'unit cell' of 'protowollastonite' can be considered to be the unit from which the layers parallel to $b c$ (Fig. 4) are developed by the $b c$ lattice net. The space-group twinning operations to produce the monoclinic and triclinic unit cells are then nothing more than the two ways of stacking these layers together (taking left- and right-handed triclinic stack- 
ing to be equivalent). It seems an unnecessary complication to formalize this with new symbols and nomenclature which do little more than obscure the physical basis of the phenomena.

I wish to thank Dr H. G. Midgley for providing the material; Prof. J.D. Bernal for his encouragement and advice; Dr F.M. Lea for permission to publish the results of work which was partly carried out under a programme of extra-mural research for the Building Research Board; and Dr H. F. W. Taylor for assistance in the early stages of the work.

\section{References}

BarnICK, A. W. (1936). Mitt. K.-Wilh.-Inst. Silikatforsch. No. 172. (Abstracted in Strukturbericht, 4, 207.)

Buerger, M. J. (1945). Amer. Min. 30, 469.

Ewald, P. P. (1940). Proc. Phys. Soc. 52, 167.

Iто, T. (1950). X-ray Studies on Polymorphism. Tokyo: Maruzen.

Peacock, M. A. (1935a). Amer. J. Sci. 30, 495.

Peacock, M. A. (1935b). Z. Krystallogr. 90, 97.

Warren, B. E. \& Biscoe, J. (1931). Z. Krystallogr. 80, 391.

WILSON, A.J.C. (1949). X-ray Optics, p. 49. London: Methuen.

Acta Cryst. (1953). 6, 825

\title{
The Multiple-Film Technique: the Effect of Angle of Incidence on the Correlating Factor
}

\author{
By G.J. BULleN \\ Department of Chemical Crystallography, University College, London W.C. 1, England
}

(Received 25 May 1953 and in revised form 17 June 1953)

\begin{abstract}
It is noted that the correlating factor for the multiple-film technique depends on the angle of incidence of the diffracted beam on the film and so has not the same value for all reflexions. The variation of the factor is discussed in detail for oscillation photographs recorded on cylindrical films with the axis of the cylinder as the axis of rotation of the crystal, the factor being constant for all reflexions on the same layer line. Methods of using spots on all layer lines, in an averaging process for finding the factor for each layer, are suggested. The treatment is also applicable to the method of interleaving films with metal foil when using Mo $K$ radiation.
\end{abstract}

The multiple-film technique first described by Robertson (1943) is nowadays widely used for the measurement of intensities by the visual method. The intensities of the spots on the second and succeeding films are related to those of the spots on the first film by a correlating factor for which Robertson gave a value of about 2 , for $\mathrm{Cu} K \alpha$ radiation at normal incidence. The variation, with angle of incidence of the diffracted beam on the film, of absorption and photographic action in a single film, as applying to intensity measurements, has been considered by Cox \& Shaw (1930) and, more recently, by Whittaker (1953). Whereas the variation of photographic action does not affect the multiple-film technique, the variation of absorption does, causing a change in the correlating factor.*

To discuss this change, let us consider an oscillation photograph recorded on cylindrical films with the axis of the cylinder as the axis of rotation of the crystal. If $I_{0}$ is the intensity recorded on the first (i.e. nearest to crystal) film and $I$ that on the second $\left(I_{0}\right.$ and $I$ being within the range of reasonably accurate intensity measurement, so that reflexions giving either very strong or imperceptible blackening of

* Note added in proof, 24 September 1953.- Since the sub. mission of this paper, this effect has been mentioned by Qurashi (1953). either film must be neglected), we have, using double emulsion films,

$$
\left(I / I_{0}\right)_{n}=\exp \left[-\left(2 \mu_{e} t_{e}+\mu_{b} t_{b}\right) \sec \nu_{n}\right],
$$

where the subscript $n$ denotes the $n$th layer line; $\mu_{e}$ and $\mu_{b}$ are the linear absorption coefficients of the emulsion and base respectively; $t_{e}$ and $t_{b}$ are the thicknesses of one side of the emulsion and of the base respectively; and $v_{n}$ is the inclination of the diffracted beam to the equatorial layer.

For the zero layer,

$$
\left(I / I_{0}\right)_{0}=\exp \left[-\left(2 \mu_{e} t_{e}+\mu_{b} t_{b}\right)\right],
$$

and thus, inverting,

$$
\left(I_{0} / I\right)_{n}=\left(I_{0} / I\right)_{0}^{\sec v_{n}}=\left(I_{0} / I\right)_{0}^{\alpha},
$$

where $\alpha=\sec \nu_{n}=\left(1-\zeta^{2}\right)^{-\frac{1}{2}}$.

Let $\left(I_{0} / I\right)_{n}=R_{n}$, the correlating factor for the $n$th layer.

Then

$$
R_{n}=R_{0}^{\alpha} .
$$

The effect is considerable for high layer lines, as $\alpha$ increases to 1.7 when $\zeta=0.8$, and is by no means negligible even for lower layers. If the intensity measurements are to be at all accurate, it must be taken into account. 